\title{
Fixed and dilated pupils after trauma, stroke, and previous intracranial surgery: management and outcome
}

\author{
H Clusmann, C Schaller, J Schramm
}

\begin{abstract}
Objectives-To clarify whether different causative events (trauma, stroke, intracranial surgery), time of intervention, and treatment mode influence outcome, patients with fixed and dilated pupils (FDPs) in a prospective neurosurgical series were evaluated.
\end{abstract}

Methods-Ninety nine consecutive patients who presented with or developed one or two FDPs, were split into three groups according to the respective aetiology: 46 patients had a trauma, 41 patients a stroke (subarachnoid or intracerebral haemorrhage), and 12 patients had undergone previous elective intracranial surgery. Appropriate therapy was performed depending on the CT findings. Outcome was classified according to the Glasgow outcome scale (GOS).

Results-Overall mortality was $75 \%$. In $15 \%$ outcome was unfavourable (GOS 2 and 3 ), and in $10 \%$ favourable (GOS 4,5 ) at 24 month follow up. No differences in outcome were found between trauma, stroke, and postelective surgery groups. Unilaterally FDP was associated with a better chance of survival $(46 \% v 13 \%$; p $<0.01)$. Age did not correlate with survival, but younger survivors had a significantly better outcome. Patients in whom an intracranial mass was removed surgically had a $42 \%$ survival rate, compared with $8 \%$ with conservative treatment $(\mathbf{p}<0.01)$. Patients with a shorter delay from FDPs to intervention had a better chance of recovery after trauma and previous intracranial surgery $(p<0.05)$. No patient survived better than a vegetative state, if previous FDPs did not become reactive shortly after therapy. If both pupils became reactive on therapy, the chance of survival was $62 \%$. Of these survivors $42 \%$ had a favourable outcome. Conclusion-Bilateral restoration of pupillary reactivity shortly after therapy is crucial for survival. Surgical evacuation of an intracranial mass, unilateral FDPs, early intervention, and younger age are related to better chances of survival or recovery. The prognosis of patients with FDPs after trauma, stroke, and previous elective intracranial surgery is similar. (f Neurol Neurosurg Psychiatry 2001;71:175-181)

Keywords: pupil; herniation; complication; prognosis
Fixed and dilated pupils in comatose patients are well known to be related to a poor prognosis, especially when present bilaterally. ${ }^{1-5}$ If not caused by local trauma or drug action, this symptom indicates injury or compression of the third cranial nerve and the upper brain stem, mainly caused by an extending intracranial mass lesion or by diffuse brain injury..$^{6-12} \mathrm{~A}$ recently suggested alternative or additional mechanism for mydriasis and brain stem symptomatology may be brain stem ischaemia. ${ }^{13}$

Due to modern emergency guidelines nearly all patients with a history of head trauma or stroke with severe impairment of consciousness are sedated, intubated, and ventilated at the emergency site. On admission to hospital most of these patients are therefore not fully assessable, so that pupillary examination remains a major tool for clinical evaluation. The finding of unilaterally or bilaterally fixed and dilated pupils (FDPs) indicates an emergency situation, and decisions on diagnostic and therapeutic procedures are to be made quickly, mainly on the basis of the personal experience of the neurosurgeons on duty. The situation is even more difficult if the neurosurgeon is contacted by telephone from a distant hospital with limited diagnostic resources. On such occasions the pupillary finding may be the only reliable symptom to be reported. Although prognostic factors after severe head injury have been widely studied, ${ }^{514-16}$ there is less information in the literature on the efficacy of neurosurgical therapy of any kind on outcome in the presence of FDPs. Moreover, the impact of different underlying causes is unclear-for example, trauma, stroke, or FDP occurring after elective intracranial surgery, the FDP indicating rebleeding or postoperative brain oedema. With socioeconomic aspects and limited resources in mind it would be helpful to know more about predictive factors for the outcome of patients with FDPs. Therefore, the goal of this study was to provide additional data for decision making in the treatment of patients presenting with FDPs due to cerebral herniation.

\section{Methods}

PATIENTS AND EXCLUSION CRITERIA

This consecutive series consisted of 99 patients (51 female, 48 male), mean age 48.5 years (range 0.2-87 years) presenting with or developing unilateral or bilateral FDPs due to an intracranial mass lesion. All such patients admitted between January 1995 and September 1996 to the Department of Neurosurgery, 
University Clinic, Bonn, were included in the study. Glasgow coma scale (GCS) scores were evaluated, if reliably documented by the emergency physician before pharmacological paralysis. ${ }^{17}$ The Department of Neurosurgery at Bonn provides the neurosurgical service for about 2 million people. During the period specified above 2337 intracranial procedures were performed. Patients were included if they showed at least one dilated pupil $(>5 \mathrm{~mm})$ not reactive to bright light. The term "ipsilateral" refers to the side of the intracranial lesion as documented by CT, and "contralateral" vice versa in bilateral FDPs. None of the patients showed a primarily contralateral FDP. The delay from occurrence of FDPs to initiation of therapy (surgical or conservative management) was registered, if the time of FDP onset was documented by either the emergency physician or by the house staff within a range of \pm 10 minutes. Post-treatment pupillary findings were assessed immediately after surgery or after initiation of conservative treatment. Pupils were documented as non-reactive or reactive to bright light.

Exclusion criteria were known or suspected administration of agents affecting the pupillary reaction to light, direct damage to the optic or oculomotor nerve, hypoxia from circulatory arrest, seizures, or primary lesions within the brain stem. Thus, 16 patients had to be excluded from the study.

PATIENT GROUPS AND MANAGEMENT

According to the documented causative events, patients were evaluated in three groups: trauma $(n=46)$, stroke, including intracerebral or subarachnoid haemorrhage, ischaemic stroke, etc $(\mathrm{n}=41)$, and previous elective intracranial surgery $(n=12)$. All patients were managed by standard treatment protocols for the various diagnoses set by the senior staff according to current medical knowledge. On admission, patients underwent cranial CT. Immediately thereafter the decision was made whether to perform surgery or to start conservative management. Neurointensive care was supplied for all patients. Generally, patients with brain oedema, cerebral contusions, subarachnoid haemorrhage (SAH), and Hunt and Hess $\mathrm{V}$ were treated conservatively. ${ }^{18}$ This included the placement of external ventriculostomy devices for intracranial pressure monitoring and CSF drainage. On rare instances $(n=4)$ craniotomy and lobectomy were performed in patients with brain oedema or ischaemic stroke. Extra-axial (subdural and epidural) haematomas were generally removed. Nondominant lobar intracerebral haematomas were removed. All postoperative rebleedings were operated on. Forty nine patients were

Table 1 Demographic data

\begin{tabular}{lllll}
\hline & \multicolumn{2}{l}{ Patients } & & \\
\cline { 2 - 3 } & $n$ & Sex & Age mean/range $(y)$ & $\begin{array}{l}\text { Fixed dilated pupils } \\
\text { unilateral/bilateral }\end{array}$ \\
\hline Trauma & 46 & $27 \mathrm{M} / 19 \mathrm{~F}$ & $43.7 / 0.2-87$ & $20(43.5 \%) / 26(56.5 \%)$ \\
Stroke & 41 & $18 \mathrm{M} / 23 \mathrm{~F}$ & $52.5 / 8-80$ & $11(26.8 \%) / 30(73.2 \%)$ \\
Postelective & 12 & $3 \mathrm{M} / 9 \mathrm{~F}$ & $53.1 / 29-79$ & $6(50.0 \%) / 6(50.0 \%)$ \\
All & 99 & $48 \mathrm{M} / 51 \mathrm{~F}$ & $48.5 / 0.2-87$ & $37(37.4 \%) / 62(62.6 \%)$ \\
\hline
\end{tabular}

treated surgically (25 men, 24 women, mean age 49.1 years), the other 50 (23 men, 27 women, mean age 48.0 years) received conservative treatment. This study did not interfere with patient management according to generally accepted standard guidelines.

FOLLOW UP AND DATA ANALYSIS

Follow up of the surviving patients was documented at 3, 12, and at 24 months after the event according to the Glasgow outcome scale (GOS): GOS 1=dead, GOS 2=persistent vegetative state, GOS $3=$ severe disability, dependent, GOS 4=moderate disability but independent, GOS 5=full recovery or minimal disability. ${ }^{19}$ If patients survived, favourable outcome was assumed in GOS 5 and 4, unfavourable in GOS 3 and 2. Patient examinations as outpatients or standardised telephone interviews with the patients or their caregivers served as information sources.

A $\chi^{2}$ test and a two tailed Student's $t$ test were applied for statistical analysis, a $p$ value $\leqslant 0.05$ being significant. Due to the few patients with good recovery, statistical analysis was not applicable to all aspects ot this study.

\section{Results}

The overall outcome in this study was as follows: 74 died (GOS 1, 75\%), five were vegetative (GOS 2, 5\%), 10 were severely disabled (GOS 3, 10\%), eight were independent (GOS $4,8 \%$ ), and two patients made a full recovery (GOS 5, 2\%) at 24 months after the initial event. Patients presenting with a unilateral FDP had a $46 \%$ chance of survival, whereas the rate was $13 \%$ with bilateral FDPs $(p<0.01)$. There were no significant differences between the three subgroups for outcome.

TRAUMA GROUP

The trauma group comprised 46 patients; for demographic data see table 1. Glasgow coma scale scores at the emergency site were available for 34 patients $(73.9 \%)$ with a median score of 5 (range 3-15). On admission, 41 patients $(89.1 \%)$ were pharmacologically paralysed and thus unassessable for GCS and neurological status.

The initial cerebral CT disclosed, as dominant findings, 19 acute subdural haematomas, 11 contusions, 10 cases of diffuse brain oedema, five epidural haematomas, and one haemorrhagic brain contusion associated with systemic coagulopathy.

The delay with an ipsilateral FDP before initiation of any treatment in the hospital and the respective outcome is shown in figure $1 \mathrm{~A}$. Delay could be determined for 30 patients with trauma. Of these, 16 patients received treatment with a delay of less than 100 minutes, and 14 had longer delays. The rate of survival with GOS better than 2 was $31.3 \%$ in the subgroup with shorter delay $(n=5)$, and $14.3 \%$ in those with longer delay $(n=2)$ (NS). The only survivor with bilateral FDPs and favourable outcome had a contralateral FDP for only $15 \mathrm{~min}$ utes. In patients with longer delay, this was generally due to preclinical reasons and delayed admission to the neurosurgical unit. 

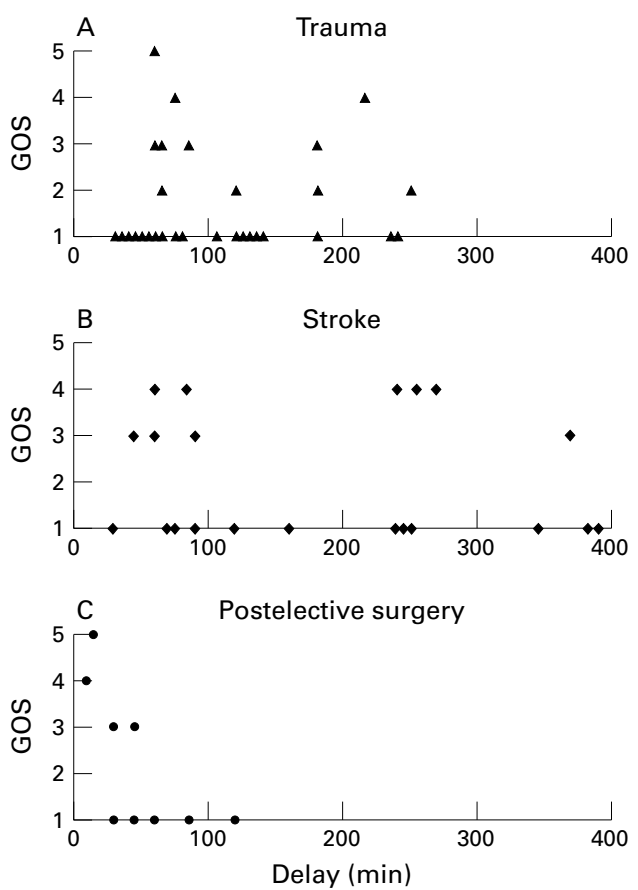

Figure 1 Delay between onset of FDP ipsilateral to the intracranial lesion and start of therapy with respect to outcome at 2 year follow up. GOS=Glasgow outcome scale. (A) trauma; (B) stroke; and (C) previous elective intracranial surgery.

Overall mortality in the trauma group was $60.0 \%$ in 20 patients with a unilateral FDP and $84.6 \%$ in 26 patients with bilateral FDPs. A favourable outcome was found in two patients $(10,0 \%)$ and one patient $(3.8 \%)$, respectively (table 2). Table 3 summarises pathology and

Table 2 Outcome at 2 year follow up with respect to unilateral or bilateral FDPs in the study population, and in the three subgroups: trauma, stroke, and post elective intracranial surgery

\begin{tabular}{lcccl}
\hline & $\begin{array}{c}\text { Patients } \\
(n)\end{array}$ & $\begin{array}{l}\text { Dead } \\
\text { GOS 1 (\%) }\end{array}$ & $\begin{array}{l}\text { Unfavourable } \\
\text { GOS 2, 3 (\%) }\end{array}$ & $\begin{array}{l}\text { Favourable } \\
\text { GOS 4, 5 (\%) }\end{array}$ \\
\hline Unilateral FDP & 37 & $20(54)$ & $11(30)$ & $6(16)$ \\
$\quad$ Trauma & 20 & $12(60.0)$ & $6(30.0)$ & $2(10.0)$ \\
Stroke & 11 & $5(45.5)$ & $4(36.4)$ & $2(18.1)$ \\
Postelective & 6 & $3(50.0)$ & $1(16.7)$ & $2(33.3)$ \\
Bilateral FDP & 62 & $55(87)$ & $4(6.5)$ & $4(6.5)$ \\
$\quad$ Trauma & 26 & $22(84.6)$ & $3(11.6)$ & $1(3.8)$ \\
Stroke & 30 & $27(90.0)$ & $-1(16.7$ & - \\
Post-elective & 6 & $5(83.3)$ & $1(10.0)$ \\
\hline
\end{tabular}

GOS $=$ Glasgow outcome scale $; \mathrm{FDP}=$ fixed and dilated pupil.

Table 3 Diagnoses and outcome at 2 year follow up

\begin{tabular}{lcccc}
\hline & $\begin{array}{c}\text { Patients } \\
(n)\end{array}$ & $\begin{array}{l}\text { Dead } \\
\text { GOS 1 (\%) }\end{array}$ & $\begin{array}{l}\text { Unfavourable } \\
\text { GOS 2, 3 (\%) }\end{array}$ & $\begin{array}{l}\text { Favourable } \\
\text { GOS 4, 5 (\%) }\end{array}$ \\
\hline Trauma & 46 & $34(73.9)$ & $9(19.6)$ & $3(6.5)$ \\
EDH & 5 & $1(20)$ & $2(40)$ & $2(40)$ \\
ASDH & 19 & $14(73.7)$ & $4(21.1)$ & $1(5.3)$ \\
CNT/oedema & 22 & $19(86.4)$ & $3(13.6)$ & - \\
Stroke & 41 & $32(78)$ & $4(9.8)$ & $5(12.2)$ \\
SAH & 21 & $18(85.7)$ & $1(4.8)$ & $2(9.5)$ \\
ICH & 14 & $9(64.3)$ & $3(21.4)$ & $2(14.3)$ \\
Other & 6 & $5(84)$ & - & $1(16)$ \\
Postelective & 12 & $8(66.6)$ & $2(16.7)$ & $2(16.7)$ \\
ICH & 7 & $5(71.4)$ & $1(14.3)$ & $1(14.3)$ \\
Oedema/other & 5 & $3(60)$ & $1(20)$ & $10(10.1)$ \\
All & 99 & $74(74.7)$ & $15(15.2)$ & $10)$ \\
\hline
\end{tabular}

GOS=Glasgow outcome scale; $\mathrm{EDH}=$ epidural haematoma; $\mathrm{ASDH}=$ acute subdural haematoma; $\mathrm{CNT}=$ contusion; $\mathrm{ICH}=$ intracerebral haemorrhage; $\mathrm{SAH}=$ subarachnoid haemorrhage, comprises acute SAH, SAH plus ICH, and sequelae of SAH as rebleeding and infarction due to vasospasm. the respective outcome. Except for one surprisingly good outcome (GOS 4) in a 75 year old man with an acute subdural haematoma, patients with a good outcome had extradural haematomas and were younger (mean age 22.5 years) compared with the unfavourable outcome group with a mean age of 45.6 years. The mean age of all survivors was 44.0 years, and 44.3 years for the patients who did not survive (NS). The mortality was $94.7 \%$ with conservative treatment, and $59.3 \%$ with surgery $(\mathrm{p}<0.05$, table 4$)$.

STROKE

The subgroup of patients presenting with one or two FDPs after stroke consisted of 41 patients (table 1). In this group a higher percentage of bilateral FDPs was found ( $n=30$, $73.2 \%$ ). Glasgow coma scale scores were available for 20 patients $(48.7 \%)$, with a median score of 5 (range 3-15). However, most patients were under deep sedation on admission. The underlying causes of stroke were 21 subarachnoid haemorrhages, 14 intracerebral haematomas, three MCA infarctions, one generalised brain oedema with sudden onset due to renal failure, one venous sinus thrombosis, and one acute pneumococcal meningitis with hydrocephalus. In $29(70.7 \%)$ of the patients the development of FDPs was the initial finding. Twelve $(29.3 \%)$ patients of this group developed one or two FDPs later in the course of their disease. Fourteen of the 21 patients with SAHs developed FDPs early on bleeding, mainly due to concomitant intracranial haemorrhage $(n=11)$. Three of these 14 patients $(21.4 \%$; all initially Hunt and Hess IV) survived after haematoma evacuation. No patient with SAH with FDPs due to rebleeding or infarction $(n=7)$ survived.

The delay with an ipsilateral FDP before initiation of any therapy with respect to outcome, available for 21 patients, is shown in figure $1 \mathrm{~B}$. The mean delays for an ipsilateral FDP were similar for all outcome classes (200 minutes GOS 1; 164 minutes GOS 2, 3; 182 minutes GOS 4, 5). By contrast, for the contralateral FDP the mean delay was shorter in three survivors (61(SD 15) minutes) than in seven non-survivors (181(SD 136) minutes) $(\mathrm{p}<0.05)$. As in the trauma group, long delays were mainly caused by delayed admission.

Overall mortality in the stroke group was $78.0 \%: 45.4 \%$ in patients with a unilateral FDP $(n=11)$ and $90.0 \%$ with bilateral FDPs $(n=30)(p<0.05)$. No patient survived in a vegetative state and no patient had a full recovery. A favourable outcome was registered in $18.2 \%$ with unilateral and $10.0 \%$ with bilateral FDPs (NS) (table 2). Underlying diseases and the respective outcome at 2 year follow up are summarised in table 3. All survivors with FDPs due to intracranial haemorrhage were treated surgically, as well as the patient with a favourable outcome after primary MCA infarction. The mean age of survivors was 46.9 years, and 54.1 years for the patients who died (NS). Patients with a favourable outcome were significantly younger than survivors with unfavourable courses $(37.6 v 58.0$ years $)(p<0.05)$. 
Table 4 Outcome at 2 year follow up with surgical or conservative treatment in the three subgroups: trauma, stroke, postelective intracranial surgery

\begin{tabular}{|c|c|c|c|c|c|c|}
\hline & \multicolumn{3}{|c|}{ Surgery $(n=49)$} & \multicolumn{3}{|c|}{ Conservative $(n=50)$} \\
\hline & Trauma & Stroke & Postelective & Trauma & Stroke & Postelective \\
\hline GOS 1 & 16 & 7 & 5 & 18 & 25 & 3 \\
\hline GOS 2 & 4 & - & - & 1 & - & - \\
\hline GOS 3 & 4 & 4 & 1 & - & - & 1 \\
\hline GOS 4 & 2 & 4 & - & - & 1 & 1 \\
\hline GOS 5 & 1 & - & 1 & - & - & - \\
\hline
\end{tabular}

GOS=Glasgow outcome scale.

The mortality was $46.7 \%$ in the surgically treated subgroup and $96.2 \%$ with non-surgical treatment $(\mathrm{p}<0.01)($ table 4$)$.

PREVIOUS ELECTIVE INTRACRANIAL SURGERY

Thirteen patients developed one or two FDPs after previous elective intracranial surgerythat is, $0.56 \%$ of all 2337 intracranial procedures performed during the study period (21 months) in the Department of Neurosurgery at Bonn (table 1). One of these 13 patients had to be excluded from the study because he developed a coagulopathy resulting in a postoperative diffuse haemorrhage within the upper brain stem. Previous elective surgery was performed for meningeomas (five patients: three brain oedema, two rebleeding), two pituitary adenomas (one rebleeding, one peracute pneumococcal meningitis with hydrocephalus), one arteriovenous malfunction (oedema), and four other entities (all rebleeding). Treatment was recraniotomy in all seven cases of rebleeding (mortality $71.4 \%$ ) and conservative including ventricular drainage for the others ( $\mathrm{n}=5$, mortality $60.0 \%$ ) (NS). By contrast with postoperative haemorrhage, patients with oedema showed a higher rate of bilateral occurrence of FDPs ( $80 \% v 38 \%$ ).

The delay with persisting ipsilateral FDP before treatment and the respective outcome is

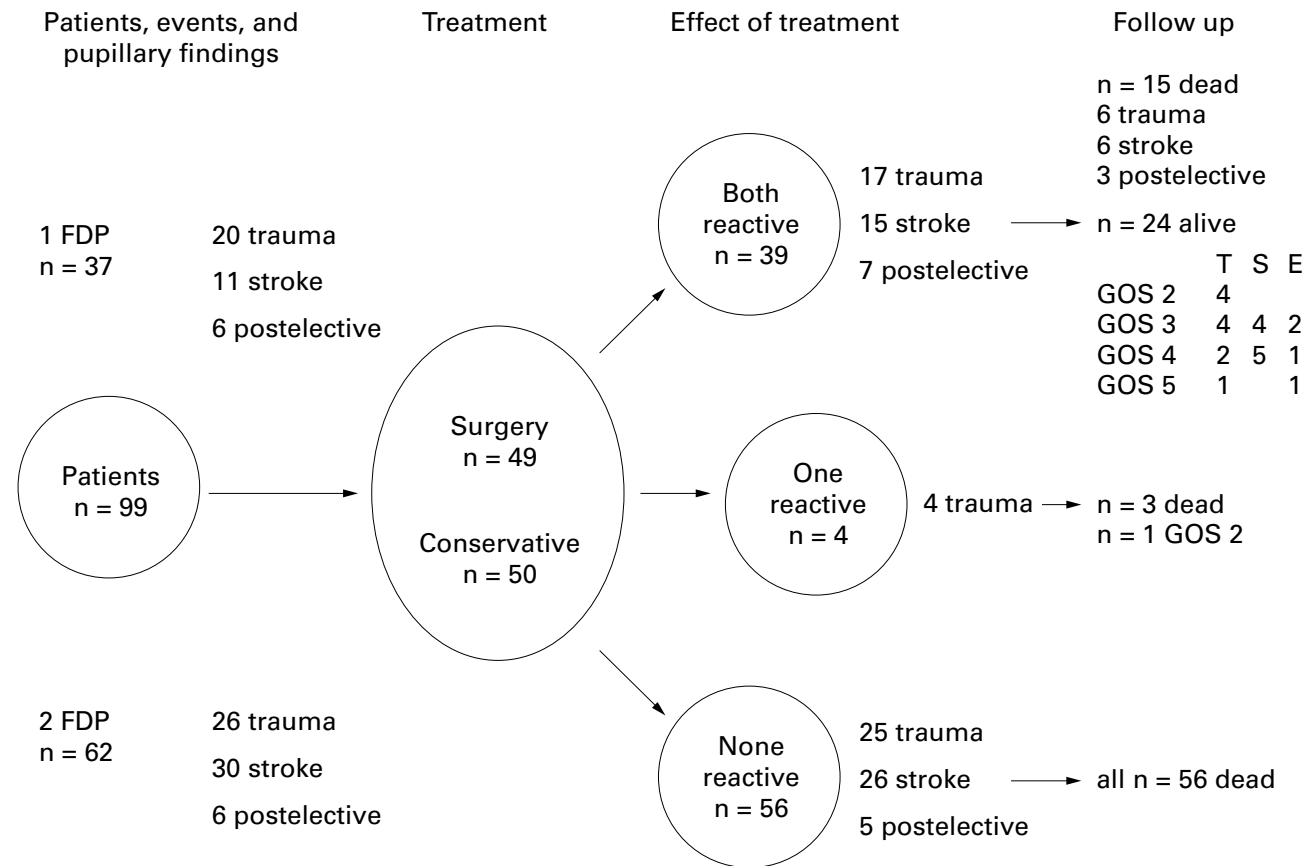

Figure 2 Overview of initial pupillary findings, management, and outcome at 2 year follow up of the whole series with respect to the efficacy of treatment (immediate post-treatment pupillary status). GOS=Glasgow outcome scale; FDP=fixed and dilated pupil; $T=$ trauma; $S=$ stroke; $E=$ postelective surgery.

outlined in figure $1 \mathrm{C}$. Delay was significantly shorter in this inpatient group (49 (SD 26) minutes) compared with the trauma (110 (55) minutes) and stroke groups (184 (SD 109) minutes $)(\mathrm{p}<0.01)$. In the postelective group faster therapy was significantly associated with a better outcome $(p<0.05)$ : a mean delay of 13 (SD 3) minutes was found in two patients with GOS 4, $5 \mathrm{v} 38$ (SD 8) minutes in two patients with GOS 2, $3 v 68$ (28) minutes in five patients with GOS 1. Only one patient with bilateral FDPs survived (GOS 3). Survivors had a mean age of 43.5 years, non-survivors 55.6 years (NS).

THERAPY, POST-TREATMENT PUPILLARY STATUS, AND OUTCOME

Table 4 summarises the outcome after surgical and conservative treatment. The mortality in the 49 surgically treated patients was $57.1 \%$ $(n=28)$, and $16.3 \%(n=8)$ of patients had a favourable outcome (GOS 4, 5). Mortality was $92.0 \%(n=46)$ in the conservatively treated group of 50 patients and $4.0 \%(n=2)$ made a favourable recovery (GOS 4), none a full recovery $(\mathrm{p}<0.01)$.

The pupillary finding after surgery or shortly after initiation of conservative treatment correlated with survival. As shown in figure 2, no patient with persisting bilateral FDPs survived. Of the four patients with one persisting FDP after treatment, only one patient survived, in a vegetative state (GOS 2). Only if both pupils were reactive to light shortly after treatment did patients have a reasonable chance of survival and recovery. The survival rate with bilateral reactivity was $61.5 \% \quad(\mathrm{n}=24)$, being significantly better than the situation with one or two persisting FDPs $(p<0.001)$. Of the survivors, $41.7 \%(n=10)$ had a favourable outcome on follow up.

Effect of treatment

$\mathrm{n}=15 \mathrm{dead}$

6 trauma

$\mathrm{n}=24$ alive

GOS 2

GOS $3 \quad 4 \quad 4 \quad 2$

GOS $4 \quad 25$ 


\section{Discussion}

This study was designed to collect information about therapy and outcome of patients with herniation due to intracranial mass lesions. Another aspect was to analyze the influence of causative events, delay, diagnoses, and mode of treatment on patient outcome. A total of $46.5 \%$ of the patients presented with FDPs after trauma, and $41.5 \%$ of the patients developed FDPs after stroke, mainly subarachnoid or intracerebral haemorrhage. In $12 \%$ of patients presenting with FDPs this occurred after elective intracranial surgery, thereby accounting for $0.56 \%$ of all intracranial procedures during the specified period. This accords with published data on complications of intracranial surgery. ${ }^{20-22}$ Many studies have evaluated management and outcome of patients with craniotomy for intracranial mass lesions in severe head injury. ${ }^{1623}$ Several predictive models have been established. ${ }^{24-27}$ One study exclusively dealt with the outcome of patients with traumatic haematomas and bilateral FDPs. Less is known about the impact of development of fixed and dilated pupils in non-traumatic patients with strokes of different underlying causes. $^{3} 182829$ The least information is available for FDPs developing after previous elective intracranial surgery. ${ }^{20-22} 30$

The results of our study generally support the well established poor prognosis in patients with ongoing brain herniation, as indicated by fixed and dilated pupils. ${ }^{57141629}$ In patients with trauma and postelective patients, the rate of bilateral FDPs was $56.5 \%$ and $53.8 \%$, whereas in the stroke group $73.2 \%$ presented with bilateral FDPs. As expected, patients with a unilateral FDP had a significantly better survival rate $(46.0 \%)$ compared with those with bilateral FDPs $(13.0 \%)$. Sakas et al reviewed the mortality in patients with trauma and transtentorial herniation. In published series (1972-91) with more than 20 patients, the range of mortality varied between $57 \%$ and $90 \% .^{5}$ In our study, no relevant changes for GOS scores occurred after 12 months, so that 24 months was considered a sufficient follow up.

\section{PATIENT AGE}

Young age is well known to be a major predictive factor for worthwhile recovery after head trauma and stroke. ${ }^{5162731}$ There were no statistical differences between the mean ages of patients in the three subgroups, although the patients with trauma were somewhat younger with a mean age of 43.7 years when compared with 52.5 and 51.8 years in the other groups. For survival versus non-survival, there was no difference in the mean ages in the trauma group. Only a non-significant tendency towards younger survivors occurred in the stroke group and in the postelective group. A more striking difference was found within the group of survivors itself. Patients with a favourable recovery after trauma had a mean age of 22.5 years, after stroke 37.6 years, whereas the ages were 45.6 years (trauma) and 58.0 years (stroke) in survivors with an unfavourable course. Our data indicate that age is a critical factor for survival and regenerative potential, in our study more striking for quality of survival than for survival itself.

DIAGNOSES, TREATMENT, AND OUTCOME

In the trauma group acute subdural haematomas were the cause of FDPs in $41.0 \%$, with a mortality of $73.7 \%$, compared with only $20.0 \%$ mortality with epidural haematomas. The prognosis for FDPs on brain contusions and global brain oedema was even worse, with a mortality of $91.7 \%$ and $80.0 \%$, respectively and no patient had a favourable outcome. The probability of a favourable outcome was $40.0 \%$ with epidural haematomas, but only $5.3 \%$ with acute subdural haematomas. The poor prognosis associated with acute subdural haematoma is well established. ${ }^{10}{ }^{26}$ It is interesting to note that the outcome in patients herniating from epidural haematomas is death or unfavourable in $60 \%$ of cases in this series. In all patients with epidural haematomas, time was unnecessarily lost by misjudgement of nonprofessionals or by postponed and consecutive late transfer to the neurosurgical unit.

Subarachnoid haemorrhage and its complications such as vasospasm and infarction accounted for $51.2 \%$ of FDPs in the stroke group. Mortality was $78.6 \%$ in the subpopulation presenting with FDPs as an initial finding. No patient survived with FDPs due to vasospasm and infarction. The prognosis for patients with FDPs due to intracranial haemorrhage or primary infarction was only somewhat better (mortality $64.3 \%$ and $66.7 \%$ ). In the stroke group no patient survived with conservative treatment. However, it is necessary to be aware of the study bias in this respect, due to internal therapeutic guidelines-for example, patients with ischaemic strokes due to vasospasm, ischaemic hemispherical strokes on the dominant side etc were generally not considered surgical candidates.

Of twelve patients developing FDPs after previous elective intracranial surgery this was in seven cases $(58.3 \%)$ due to a rebleeding. All of these haematomas were removed surgically; nevertheless, five patients died (71.4\%). Most rebleedings leading to FDPs in this series occurred after high risk procedures - for example, the 26th resection of a spheno-orbital meningioma or the operation of a giant cerebellar haemangioblastoma. The occurrence of FDPs due to brain oedema occurred mainly after resection of extensive meningiomas with already preoperatively significant peritumorous brain swelling. The rate of postoperative haemorrhages was reported to range between $0.8 \%$ and $3.9 \%,{ }^{20-22}$ but criteria and classifications differed between these studies. Mortality was reported to be around 30\%, which corresponds well to the rate found with FDPs in our study. Moreover, surgery for meningiomas was generally the most frequent cause for rebleeding. ${ }^{20}{ }^{21}$ It has to be noted that due to postoperative patient monitoring on the neurosurgical intensive care unit, most complications after surgery were detected and treated before the patients developed FDPs. In some 
cases problems occurred in the very early postoperative period, when patients were still under sedation. The occurrence of FDPs despite meticulous countermeasures may account for the fact that the prognosis of inpatients, once they developed FDPs, is nearly as bad as in the trauma and stroke groups. ${ }^{2122} 30$

DELAY AND MECHANISMS OF FDPS

The delay between manifestation of FDPs and initiation of therapy was analyzed, because it is a measure for evaluating the process of transportation, establishing a diagnosis, and initiating treatment. Sakas et al reported a range of 1-9 hours delay for surgical treatment of bilateral FDPs in traumatic brain injury and found 3 hours to be a critical value for survival. ${ }^{5}$ In the trauma and stroke groups, the mean delays were virtually equal in all outcome classes. However, the mean delay was shorter in the trauma group (106 minutes) than after stroke (184 minutes). This difference may be due to the fact that more immediate attention is directed towards victims of accidents and that many of these patients are primarily transported to a neurosurgical unit. As can be derived from figure 1, many patients with trauma or stroke were treated within the first hour. In both groups there is a second peak of longer latencies, due to late transfer to the neurosurgical unit, especially from rural areas. It can be assumed that prognosis could generally be somewhat better with shorter delays in the trauma and stroke groups. ${ }^{523}$ Nevertheless, early intervention does not guarantee survival or favourable outcome. ${ }^{23}$ After elective surgery delay was shorter and earlier treated patients seemed to do better, although the better general outcome was only a trend compared with the other groups.

A unilateral FDP is thought to be due to third cranial nerve compression, which does not itself lead to irreversible brain damage. On the other hand, bilateral FDPs are thought to be already associated with alterations in the upper brain stem. ${ }^{3}{ }^{12}$ It has recently been shown that blood flow in the brain stem is critically reduced in patients with FDPs, probably due to compromise of perforating vessels from the basilar artery. ${ }^{13}$ There seems to be a good correlation between recovery and preserved brain stem perfusion. ${ }^{13}$ It can be speculated that in many cases at least early stages of unilateral FDPs and persisting bilateral FDPs may be similar symptoms with different underlying pathophysiology. These data may explain differences in outcome with unilateral and bilateral FDPs and account for possible initial contralateral pupil enlargement as well as for difficulties in outcome prediction with purely clinical information. ${ }^{28}$ Another proposed mechanism in unilateral FDPs are the occurrence of regional changes in cerebral blood flow, whereas with bilateral FDPs a global hypoperfusion is seen. ${ }^{32}$

THERAPY, POST-TREATMENT PUPILLARY FINDINGS, AND PROGNOSIS

The choice of treatment was dependent on the respective diagnosis. Therefore, treatment modes cannot be independently analyzed. Nevertheless, it is worthwhile to look at this feature, because choice of treatment shows information about the patient's condition and the options available to the neurosurgeon. Generally, whenever it seemed possible to remove a space occupying lesion, all attempts were made to do so. As summarised in table 5, the prognosis after FDPs was much better when a surgical procedure was considered adequate. Conservative treatment only rarely led to survival $(8 \%)$ or favourable outcome $(4 \%)$. A circumscribed space occupying intracranial lesion and its surgical removal gave a $42 \%$ chance of survival.

Outcome was clearly dependent on the immediate post-treatment pupillary finding (fig 2). No patient survived with bilateral nonreactive pupils immediately after treatment. No patient survived better than a vegetative state with only one pupil reactive to light after treatment. Much better results were found when both pupils showed reactivity to light immediately after therapy. Of these patients $61.5 \%$ survived and $25.6 \%$ had a favourable outcome. With survival, a favourable outcome was least probable in the trauma group $(27.3 \%)$, but better after stroke $(55.6 \%)$ and after previous intracranial surgery $(50.0 \%)$. The impact of early postoperative restoration of the pupil's reaction to light is a matter of discussion. Sakas et al only found some tendency for better recovery with early restoration of pupil's reactivity, ${ }^{5}$ whereas Andrews and Pitts found this to be absolutely necessary for functional recovery in patients with trauma. ${ }^{16}$ This second finding is supported by our data for patients with trauma, stroke, or previous intracranial surgery.

In summary, examination of the pupil's reaction to light is an important feature in comatose patients and in many cases with pharmacological paralysis after trauma or stroke. Early after intracranial surgery it is an integral part of neurological assessment. Once showing unilateral or bilateral FDPs, the most important factors associated with survival were: only a unilateral FDP, localised space occupying lesion and consecutive surgical therapy, and bilaterally positive pupil's reaction to light immediately after treatment. In patients with bilateral FDPs favourable outcomes were only seen with delays of less than 90 minutes. No patient survived better than a vegetative state with only one pupil showing reaction to light immediately after treatment and no patient survived with persisting bilateral FDPs.

1 Becker DP, Miller JD, Ward JD, et al. The outcome from severe head injury with early diagnosis and intensive management. F Neurosurg 1977; 47:491-502.

2 Andrews BT, Pitts LH, Lovely MP, et al. Is computed tomographic scanning necessary in patients with tentorial herniation? Results of immediate surgical exploration without computed tomography in 100 patients. Neurosurgery 1986;19:408-14.

3 Krieger D, Adams HP, Schwarz S, et al. Prognostic and clinical relevance of pupillary responses, intracranial brainstem auditory evoked potentials in comatose patients with acute supratentorial
mass lesions. Crit Care Med 1993;21:1944-50.

4 Choi SC, Barnes TY, Bullock R, et al. Temporal profile of outcomes in severe head injury. F Neurosurg 1994;81:16973. 
5 Sakas DE, Bullock MR, Teasdale GM. One-year outcome following craniotomy for traumatic hematoma in patients
with fixed dilated pupils. 7 Neurosurg $1995 ; 82: 961-5$.

6 Jefferson G. The tentorial pressure cone. Arch Neurol Psychiatry 1938;40:857.

7 Reid WL, Cone WV. The mechanism of fixed dilatation of the pupil resulting from cerebral compression. $\mathcal{F A M A}$ 1939;112:2030

8 Johnson RT, Yates PO. Clinico-pathological aspects of pressure changes at the tentorium. Acta Radiol 1956;46:242-9.

9 Hoff JT, Spetzler R, Winestock D. Head injury and early signs of tentorial herniation. a management dilemma. West f Med 1978;128:112-16.

10 Seelig JM, Greenberg RP, Becker DP, et al. Reversible brain-stem dysfunction following acute traumatic subdural hematoma. a clinical and electrophysiological study. $f \mathrm{Neu}$ rosurg 1981;55:516-23.

11 Andrews BT, Chiles BW, Olsen WL, et al. The effect of intracerebral hematoma location on the risk of brain-stem intracerebral hematoma location on the risk of brain-stem 518-22.

12 Ropper AH. The opposite pupil in herniation. Neurology 1990;40:1707-9.

13 Ritter AM, Muizelaar JP, Barnes T, et al. Brain stem blood flow, pupillary response, and outcome in patients with severe head injuries. Neurosurgery 1999;44:941-8.

14 Gutterman P, Shenken HA. Prognostic features in recovery from traumatic decerebration. $\mathcal{F}$ Neurosurg 1979;32:330-5.

15 Jennett B, Teasdale G, Braakman R, et al. Prognosis of patients with severe head injury. Neurosurgery 1979;4:2839.

16 Andrews BT, Pitts LH. Functional recovery after traumatic transtentorial herniation. Neurosurgery. 1991;29:227-31.

17 Teasdale G, Jennett B. Assessment of coma and impaired consciousness. A practical scale. Lancet 1974;ii:81-4.

18 Hunt WE, Hess RM. Surgical risk as related to time of intervention in the repair of intracranial aneurysms. $\mathcal{F} \mathrm{Neu}$ rosurg 1968;28:14-20.

19 Jennett B, Bond M. Assessment of outcome after severe brain damage. Lancet 1975;i:480-4.

20 Fukamachi A, Koizumi H, Nukui H. Postoperative intracerebral haemorrhages. A survey of computed tomographic findings after 1074 intracranial operations. Surg Neurol 1985;23:575-580.
21 Kalfas IH, Little JR. Postoperative hemorrhage: A survey of 4992 intracranial procedures. Neurosurgery 1988;23:343-7. 22 Palmer JD, Sparrow OC, Iannotti F. Postoperative hematoma. A 5-year survey and identification of avoidable risk factors. Neurosurgery 1994;35:1061-4.

23 Seelig JM, Becker DP, Miller JD, et al. Traumatic acute subdural hematoma. Major mortality reduction in comatose patients treated within 4 hours. N Engl f Med 1981;304: patients treat $1511-18$.

24 Jennett B, Teasdale G, Braakman R. Predicting outcome in individual patients after severe head injury. Lancet 1976; 1031-4.

25 Schynoll W, Overton D, Krome R, et al. A prospective study to identify high-yield criteria associated with acute intracranial computed tomography findings in headinjured patients. Am f Emerg Med 1993;11:321-6.

26 Yanaka K, Kamezaki T, Yamada T, et al. Acute subdural hematoma: prediction of outcome with a linear discriminant function. Neurol Med Chir (Tokyo) 1993;33:552-8.

27 Signorini DF, Andrews PJ, Jones PA, et al. Predicting survival using simple clinical variables. a case study in traumatic brain injury. $\mathcal{F}$ Neurol Neurosurg Psychiatry 1999;66: $20-5$

28 Chen R, Sahjpaul R, Del Maestro RF,et al. Initial enlargement of the opposite pupil as a false localising sign in intraparenchymal frontal haemorrhage. $\mathcal{f}$ Neurol Neurosurg Psychiatry 1994;57:1126-8.

29 Bailes JE, Spetzler RF, Hadley MN, et al. Management morbidity and mortality of poor-grade aneurysm patients. $\mathcal{F}$ Neurosurg 1990;72:559-66.

30 Taylor WA, Thomas NW, Wellings JA, et al. Timing of postoperative intracranial hematoma development and implications for the best use of neurosurgical intensive care. $\mathscr{f} \mathrm{Neu}$ rosurg 1995;82:48-50.

31 Jamjoom A, Nelson R, Stranjalis G, et al. Outcome following surgical evacuation of traumatic intracranial haematomas in the elderly. Br f Neurosurg 1992;6:27-32.

32 Marion DW, Darby J, Yonas H. Acute cerbral blood flow changes caused by severe head injuries. 7 Neurosurg 1991;74:407-14.

\title{
Narrative Based Medicine, An Interdisciplinary Conference
}

\author{
Research, Narrative, and Practice
}

A two day conference-Monday 3rd and Tuesday 4th September 2001

\section{Homerton College, Cambridge, UK}

\section{BMF Publishing Group}

For full details contact: BMA/BMJ Conference Unit, Tavistock Square, London, WC1H 9JP Tel: +44 (0)20 7383 6819; fax: +44 (0)20 7383 6663; email: clyders@bma.org.uk.

www.quality.bmjpg.com 